\title{
INCONTINENCIA URINARIA, CÁNCER DE PRÓSTATA Y RADIOTERAPIA EXTERNA
}

\author{
Francisco Javier Celada Álvarez.
}

Servicio de Oncología Radioterápica. ERESA. Hospital General de Elche. Elche. Alicante. España.

\begin{abstract}
Resumen.- La radioterapia externa está consolidada como tratamiento radical en el cáncer próstata localizado y localmente avanzado. Además, cada vez más estudios muestran su eficacia tanto en la adyuvancia como en el rescate. Antes de elegir una opción terapéutica es importante conocer la situación basal de los pacientes y los posibles efectos secundarios del tratamiento. Para ello disponemos de diferentes cuestionarios y escalas de toxicidad. Los efectos agudos, que se dejan notar durante el tratamiento y las semanas siguientes, se deben a síntomas irritativos e inflamatorios. La toxicidad crónica, meses o años tras la radioterapia, es consecuencia de la fibrosis e isquemia, así como de alteraciones a nivel miógeno y neuronal. La incontinencia urinaria es un sintoma relativamente poco frecuente dentro de la toxicidad asociada a la radioterapia. Pero, su importante impacto en la calidad de vida de nuestros pacientes nos obliga a determinar quienes tienen más riesgo de padecerla. El antecedente de resección transuretral de próstata, antes o tras la irradiación, y la severidad de la toxicidad genito-urinaria aguda son factores riesgo para el desarrollo de incontinencia.
\end{abstract}

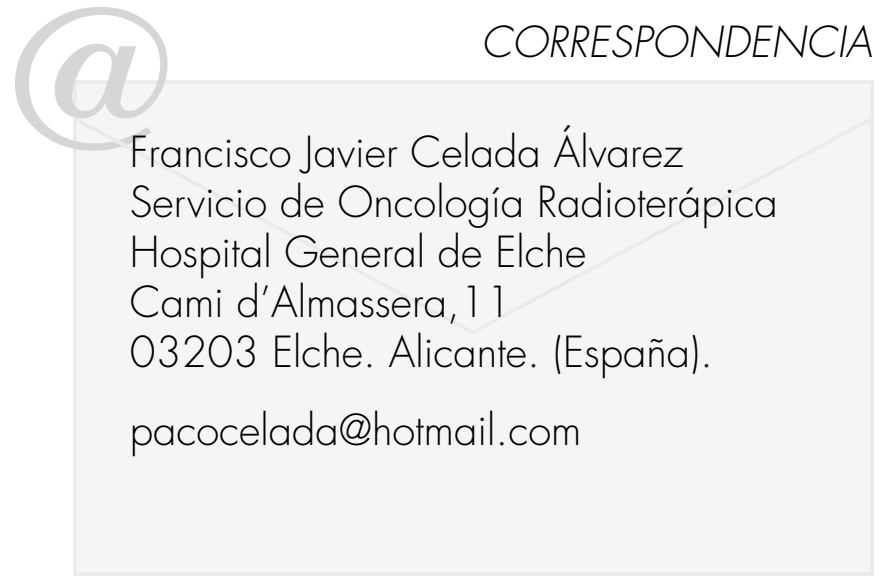

Palabras clave: Cáncer de próstata. Radioterapia. Toxicidad. Incontinencia urinaria.

Summary.- External beam radiotherapy is consolidated like radical treatment for localized and locally advanced prostate cancer. In addition, more and more studies show its effectiveness as much in postoperative as in salvage intention. Before choosing a therapeutic option, it is important to know the baseline state of the patients and the possible side effects of the treatment. In that way, different questionnaires and toxicity scales are at our disposal. Acute effects, during the treatment and the following weeks, are due to irritating and inflammatory symptoms. Chronic toxicity, months or years after radiotherapy, are consequence of the fibrosis and ischemia, as well as of alterations at the myogenic and neuronal level. Urinary incontinence is a relatively uncommon symptom among patients who have been treated with radiotherapy. But, due to the profound impact on the quality of life of our patients, it is necessary to identify patients at higher risk. Antecedent of transurethral resection of the prostate, before or after the irradiation, and severity of acute genitourinary toxicity increase the risk of developing incontinence.

Keywords: Prostate cancer. Radiotherapy. Toxicity. Urinary incontinence.

\section{INTRODUCCIÓN}

Las neoplasias de órganos situados en la pelvis son muy comunes. El cáncer de próstata en el hombre, y la patología cérvico-uterina en la mujer, ya independientemente de sus estadios, son protagonistas habituales en los servicios de Oncología Radioterápica. 
En los casos de cáncer de próstata localizado, hoy en día se disponen de diferentes abordajes terapéuticos. Los nuevos avances técnicos, especialmente en las dos últimas décadas, han puesto en boga a lo largo de todo el mundo a la radioterapia externa (RTE) y a la braquiterapia (BT). Tanto la prostatectomía radical (PR), como la RTE y la BT ofrecen resultados superponibles en cuanto a control bioquímico (10). No hay estudios randomizados que evidencien la superioridad de alguna de las opciones en control tumoral o supervivencia. Así, la opinión del primer especialista al que visita el paciente a menudo determina el tratamiento elegido (2).

En la actualidad el concepto de calidad de vida (QOL) ha de pesar a la hora de tomar las decisiones correctas. En concreto la toxicidad genito-urinaria (GU) es determinante en la QOL post-tratamiento sea cual sea la opción terapéutica. Hay varios estudios que comparan la $Q O L$ tras las tres opciones, pero la mayoría no incluye una evaluación pretratamiento (35). La QOL pre-tratamiento debería jugar un papel fundamental en la decisión a tomar, aunque en muchas ocasiones se ofrecen terapias inapropiadas (6).

Un estudio prospectivo español, que sí evaluó la QOL pre-tratamiento, demostró que hay diferencias en los perfiles de toxicidad a los 2 años. Mientras la cirugía tiene un considerable impacto negativo sobre la función sexual y la continencia, la RTE presenta un moderado efecto negativo sobre el aparato digestivo y la función sexual. La BT destaca por un ligero incremento de los síntomas irritativos (7). El riesgo de incontinencia urinaria tras RTE, se puede considerar en general muy bajo. Revisando la literatura se pueden encontrar rangos del 0 al 10\% dependiendo de la definición usada (8).

Se disponen de diferentes cuestionarios de QOL y escalas de morbilidad, cada uno de ellos con sus carencias. A pesar de los intentos por uniformar criterios, aún queda camino por recorrer en esa dirección.

Los síntoma agudos durante el tratamiento radioterápico, en general autolimitados, son fundamentalmente irritativos, pero podemos encontrar incontinencia acompañando a la urgencia en el contexto de una vejiga hiperactiva. Pero son los síntomas tardíos, en relación con fibrosis e isquemia, los que más van a marcar la QOL del paciente. La disfunción vesical, y su disminución de volumen, conllevan a la presencia de síntomas como incontinencia.

A la hora de estudiar la incontinencia urinaria post-RT hay que diferenciar según la intención del tratamiento, sea radical, adyuvante, o de rescate.

\section{FISIOPATOLOGÍA DE LA IRRADIACIÓN}

La RTE, en el caso de la patología pélvica, consiste en la transmisión de paquetes o quanta energéticos, denominados fotones, que al interaccionar con los tejidos van a inducir lesiones en los mismos. El mecanismo puede ser por acción directa, interaccionando directamente con macromoléculas como el ADN y provocando alteraciones y roturas cromosómicas, o por acción indirecta, desencadenando la radiolisis del agua del medio y así generando radicales libres y agentes oxidativos que inducen la respuesta celular. Estos defectos celulares pueden ocasionar la muerte celular directa por lisis mitocondrial, o dar lugar a la imposibilidad de división acabando en una muerte celular diferida.

Los efectos agudos se dejan sentir durante el tratamiento y en las semanas posteriores, estando en relación con cambios inflamatorios y edema a nivel de la mucosa. La pérdida de las capas más superficiales produce una denudación del epitelio (urotelio) que coincide clínicamente con la aparición de síntomas irritativos. En este momento, el tejido sano inicia una repoblación acelerada que consigue contrarrestar los efectos de la irradiación.

Los cambios crónicos, que aparecen meses o años tras la RT, se deben principalmente a daños en los tejidos vascular y conectivo. En los pequeños vasos las lesiones en su endotelio producen dilatación y formación de telangiectasias, trombosis y/o roturas de la pared. En los vasos de mayor tamaño procesos como la proliferación de la íntima, la hialinización de la capa media o la fibrosis de la adventicia producen la obstrucción del vaso. En el estroma la fibrosis es la reacción característica. La necrosis, la formación de fístulas o la sobreinfección pueden ser la consecuencia de esta hipoxia y de la fibrosis tisular. Además, como reacción se induce la neoformación de vasos muy frágiles con la hemorragia como resultado (8). También posibles alteraciones a nivel miógeno y neuronal se relacionan con la aparición de síntomas urinarios por RT. Una mucosa uretral con la sensibilidad comprometida parece relacionarse con la incontinencia urinaria (9).

Conocidos los factores radiobiológicos y moleculares, la toxicidad de los tejidos sanos (y lógicamente la respuesta tumoral) también depende de otros factores relacionados con el tratamiento. La dosis total, el fraccionamiento (dosis/sesión) y el volumen son parámetros a tener en cuenta. Las dosis máximas de tolerancia, obtenidas de la experiencia, permitían estimar la probabilidad de complicaciones en un determinado tiempo considerando la irradiación de todo el órgano con fraccionamiento convencional (10). Por ejemplo, la dosis que produce un $5 \%$ de posibilidad 
de ulceración de la vejiga a los cinco años está estimada en 65 Gy Pero el volumen irradiado también influye en la toxicidad. En la práctica actual, con la planificación virtual, se elaboran histogramas dosis-volumen que permiten establecer la dosis administrada a un volumen determinado de un órgano sin riesgo de complicaciones.

\section{CUESTIONARIOS DE CALIDAD DE VIDA Y ESCALAS DE TOXICIDAD}

Tanto urólogos como oncólogos radioterápicos, conocedores de la equivalencia en cuanto a supervivencia de las diferentes armas terapéuticas, nos enfrentamos a la dificultad de aconsejar sobre la mejor opción de tratamiento. Dada la larga esperanza de vida hemos de esforzarnos por garantizar la mejor QOL posible. Para evaluar la QOL disponemos de cuestionarios como escalas estandarizadas de evaluación del aparato genito-urinario. Éstos nos permiten valorar la situación basal, la toxicidad posttratamiento y comparar los distintos esquemas de tratamiento.

Disponemos de cuestionarios de carácter genérico (como SF-36 y FACT-G), pero para patología urológica se han desarrollado otros más específicos. No todos los cuestionarios otorgan el mismo peso a los diferentes síntomas. Probablemente el más conocido es el IPSS (International Prostate Store System), adoptado por la OMS. Es un cuestionario breve, con siete preguntas sobre síntomas urinarios y una sobre
QOL global. Pero presenta un sesgo hacia los síntomas irritativos respecto a la continencia.

Un cuestionario más extenso, quizá demasiado para uso rutinario, es el EPIC (Expanded Prostate cancer Index Composite). Fue diseñado para evaluar el impacto de las distintas opciones terapéuticas en pacientes con cáncer de próstata localizado, y sí es capaz de diferenciar si el malestar en la esfera urológica se debe a síntomas irritativos/obstructivos o de incontinencia. El FACT-P (Prostate Cancer Treatment Outcome Questionnaire) es un módulo específico para pacientes con cáncer de próstata del FACT-G (1 1). Incluye doce preguntas sobre incontinencia urinaria y otros síntomas.

Probablemente el cuestionario sobre incontinencia más recomendado sería el Internationational Continence Society Male Short Form aunque sólo esta validado para hipertrofia benigna de próstata (12).

Hay numerosos estudios sobre cáncer de próstata y QOL. Se han de tener en cuenta varias consideraciones a la hora de interpretarlos. Es necesario evaluar la situación basal previa al tratamiento. Se ha comprobado que el paciente recuerda la $Q O L$ pretratamiento mejor de lo que fue en realidad. Este efecto, que empeora con la edad y mejora con el nivel educativo, es independiente del tiempo transcurrido entre el tratamiento y la entrega del cuestionario, y afecta a los estudios retrospectivos (13). Por otra parte, las tasas de incontinencia registradas en los cuestionarios suelen ser mayores que cuando el médico es el encar-

TABLA I. LA INCONTINENCIA URINARIA TARDÍA EN LAS ESCALAS DE TOXICIDAD.

\begin{tabular}{|c|c|c|c|c|c|}
\hline & GO & G1 & G2 & G3 & G4 \\
\hline RTOG/EORTC & \multicolumn{5}{|c|}{ Sin referencias a la incontinencia } \\
\hline LENT/SOMA & - & $\begin{array}{c}\text { Ocasional } \\
(<1 \mathrm{p} / \text { semana) uso } \\
\text { de pañales }\end{array}$ & $\begin{array}{c}\text { Intermitente } \\
(<1 \mathrm{p} / \text { día) uso de } \\
\text { pañales }\end{array}$ & $\begin{array}{c}\text { Persistente } \\
\text { (diario) uso de } \\
\text { pañales }\end{array}$ & $\begin{array}{l}\text { Sondaje vesical } \\
\text { permanente, } \\
\text { cistostomía o } \\
\text { cistectomía }\end{array}$ \\
\hline CTCAE v3.0 & - & $\begin{array}{l}\text { Ocasional (p.e. } \\
\text { Valsalva) uso de } \\
\text { pañales }\end{array}$ & $\begin{array}{l}\text { Espontáneo, uso } \\
\text { de pañáles } \\
\text { indicado }\end{array}$ & $\begin{array}{l}\text { Interfiere con las } \\
\text { actividades } \\
\text { diarias. Indicada } \\
\text { inervención } \\
\text { (inyecciones de } \\
\text { colágeno..) }\end{array}$ & $\begin{array}{c}\text { Indicada } \\
\text { intervención } \\
\text { quirúrgica } \\
\text { (cistostomía o } \\
\text { cistectomía) }\end{array}$ \\
\hline
\end{tabular}


gado de recogerlas. Además la entrevista telefónica puede evitar injerencias externas y facilita la respuesta sobre aspectos íntimos. Éste fue el sistema elegido para el único estudio prospectivo multicéntrico español sobre QOL y tratamiento del cáncer de próstata, ya comentado anteriormente y de interesante lectura (7).

Simplificando, el desafío técnico en la Oncología Radioterápica, consiste en administrar una dosis suficiente al volumen tumoral, destruyéndolo, pero respetando al máximo el tejido sano circundante. Por eso, desde siempre, ha habido una especial consideración hacia los posibles efectos secundarios. Para la valoración de la toxicidad disponemos de varias escalas con apartados concretos para el aparato genito-urinario (Tabla I). La escala de la RTOG-EORTC, probablemente la más usada, no incluye ni la urgencia ni la incontinencia como fenómenos tardíos (14). La escala LENT-SOMA, algo más compleja, se desarrolló para caracterizar mejor la toxicidad GU y sí recoge la incontinencia (15). El National Cancer Institute ofrece la tercera versión del CTCAE (Common Toxicity Criteria and Advese Effects) (http://ctep.cancer.gov), donde la incontinencia está presente como uno de los síntomas a valorar.

Sin duda es necesario uniformar criterios para poder comparar resultados. Por ejemplo, usar la escala de la RTOG puede llevar a perder la recogida de gran parte de la toxicidad tardía Grado 3, en especial la relacionada con la incontinencia (16).

\section{INCONTINENCIA Y RADIOTERAPIA EXTERNA RADICAL}

No hay duda que, gracias al perfeccionamiento de las técnicas quirúrgicas, las complicaciones como la incontinencia cada vez son menos frecuentes. Pero a la vez, la Oncología Radioterápica ha asistido a una evolución, una revolución, que la ha llevado de la antigua radioterapia en dos dimensiones a las tres dimensiones (RT3D, disponible a lo largo de todo el territorio nacional) y a la intensidad modulada (IMRT, implantada en muchos centros). Incluso, en un análisis retrospectivo, el año de tratamiento era factor independiente de resultado en pacientes tratados con RTE radical (17). Este progreso ha permitido el descenso en la tasa de complicaciones y la escalada de dosis, logrando así mayor control tumoral.

La mayoría de las complicaciones GU postRT están relacionadas con síntomas irritativos, especialmente en la fase aguda. La incontinencia es una complicación poco frecuente. Una revisión de 1995 Marks encontró tasas del $0-10 \%$ dependiendo de la definición usada (8). El estudio con la tasa de incon- tinencia más alta (10\%) incluía síntomas menores y transitorios como la incontinencia de estrés (18). En 1994 Perez, en un estudio sobre los factores técnicos que afectan a la morbilidad, sólo comunicó 5/738 pacientes que desarrollaran incontinencia y los relacionó con RTU previas (19). Shirpley tras estudiar a más de 2500 pacientes tratados con RT, halló una tasa del $0.9 \%(20)$. Ese mismo año Jonler publicaba tasas del $13 \%$ con pequeñas pérdidas, aunque sin evaluación previa al tratamiento (21). En publicaciones más recientes se han descrito tasas mucho más bajas, como Brabbins con una tasa de $0 \%$ de incontinencia G3 según CTCAE v2.0 (22). Un interesante artículo publicado recientemente evalúa la toxicidad $\mathrm{GU}$ tras altas dosis de RT. Notifica una toxicidad GU tardía G2 de 19\%, pero sólo un paciente con incontinencia (1\%), que posteriormente se recuperó. Eso sí, de los 7 pacientes (3\%) que desarrollaron toxicidad GU G3, 5 $(2 \%)$ fue debida a incontinencia, aunque 2 de ellos ya la presentaban previa al tratamiento (16). En cambio, en un estudio del Memorial Sloan-Kettering sobre toxicidad a los 10 años tras RTE, encontraron que, en el $95 \%$ de los pacientes con toxicidad G2, los síntomas estaban relacionados con aumento de la frecuencia y urgencia. La incontinencia que precisaba de pañales fue muy inusual (23). Un estudio orientado específicamente a la incontinencia urinaria post-RT evaluó a 1192 pacientes. Usando la escala modificada RTOG/ $S O M A$, en la que la incontinencia $G \geq 1$ supone el uso ocasional de pañales, y el grado $\geq 2$ el uso intermitente, las tasas de incontinencia a los cinco años fueron del $7 \%$ y $1.7 \%$ respectivamente (24).

Estos resultados son acordes con los publicados por Lee, que usando la misma escala encontró tasas del $1.3 \%$ para $\mathrm{G} \geq 2(25)$.

Según lo publicado, la incontinencia es poco habitual en la mayoría de las series. Pero, aún así, y especialmente debido al efecto limitante de esta complicación, debemos tenerla en consideración y ser capaces de identificar a los pacientes con factores de riesgo de desarrollarla. Varios estudios aportan datos que sugieren que los pacientes con antecedentes de resección transuretral de próstata (RTUP) tienen mayor riesgo de desarrollar incontinencia $(8,19,25-27)$. A pesar de ello, la posibilidad de sufrir incontinencia clínicamente significativa sigue siendo baja. En el estudio de Liu, 7 de 246 pacientes con historia de RTU tuvieron incontinencia $G \geq 2$ (2.85\%) (24). De nuevo estos datos concuerdan con los del Fox Chase Cancer Center (2\%) (25). Un estudio diseñado para evaluar esta relación encontró que, para los paciente irradiados con antecedentes de RTUP, la tasa de incontinencia fue del $5.4 \%$, comparada con el $1 \%$ de los que no se habían sometido a dicha técnica (26). Parece claro que la manipulación quirúrgica del cuello vesical y de la uretra está 
relacionada con los problemas de continencia. Probablemente se debe a una relativa desvascularización de la uretra tras la RTUP, y que disminuye la capacidad de la mucosa para reparar el daño subletal ocasionado por la RTE. En cualquier caso, el inicio de RTE debería demorarse al menos 4-6 semanas tras RTUP. ¿̇Y si se cambia el orden de los factores? En ocasiones, debido a los síntomas obstructivos post-RTE, es preciso la realización de una RTUP. En la serie de Liu, este hecho provoca un aumento de la tasa de incontinencia $G \geq 2$ $(8 \%)$ a los 5 años (24). Hay estudios contrapuestos en esta dirección. Patel, en una pequeñísima serie de 7 pacientes con RTU post-RTE, no comunicó ninguna complicación (28). Para Holzman, de 44 pacientes con recaída bioquímica post-RTE y RTU ulterior, 12 desarrollaron una incontinencia absoluta (29). Ahora, el defecto en la cicatrización por culpa de la RTE sería el mecanismo de acción responsable.

Es conocido que la toxicidad GU aguda tras RTE está relacionada con un aumento de los efectos crónicos $(23,30)$. Este patrón también es seguido en el caso de la incontinencia. Los pacientes que sufren toxicidad GU aguda de cualquier grado, tienen prácticamente el doble de posibilidades de desarrollar incontinencia $G \geq 2(24,25)$.

Otros factores de riesgo, como el estadio tumoral, son más discutibles. Jonler describía que los pacientes irradiados con afectación extracapsular (T3) tenían mayores tasas de incontinencia (21). Esos datos no han sido corroborados en otros estudios $(23,24)$.

Además, es lógico presuponer que una enfermedad localmente avanzada pueda comprometer el control del esfínter. También son pacientes con más riesgo de síntomas obstructivos, y por consiguiente con más posibilidades de haber necesitado una RTUP previa. Las dosis altas y los volúmenes amplios de tratamiento se ha relacionado con mayor toxicidad GU $(8,23)$. Sin embargo, en el análisis multivariante de Liu no resultan significativos para el desarrollo de incontinencia crónica. La toxicidad vesical, tipo cistitis y contractura, es la más relacionada con estos aspectos. Por otra parte, factores como la edad, diabetes mellitus, tratamiento hormonal (30) o toxicidad gastro-intestinal por RTE no son responsables de un empeoramiento de la capacidad de continencia tras RTE.

\section{INCONTINENCIA Y RADIOTERAPIA TRAS PROSTATECTOMIAA}

En el tratamiento radioterápico tras prostatectomía aún quedan muchas cuestiones por resolver. Entre ellas el momento óptimo para el inicio de tratamiento, ya sea de forma adyuvante o tras recaída, bioquímica o clínica. La RT adyuvante en pacientes pT3, con o sin márgenes afectos, reduce las recidivas locales, con un potencial impacto en la supervivencia aún no demostrado. Hay estudios Fase III que demuestran una mejoría en la supervivencia libre de recaída bioquímica o clínica $(31,32)$. Pero estos estudios no disipan todas las dudas, y para mejorar la ganancia terapéutica es necesario identificar a aquellos pacientes con mayor riesgo de enfermedad residual. Así, hoy en día el manejo de estos pacientes va desde la RT adyuvante a la observación. Como siempre, a la hora de tomar una decisión hemos de tener en cuenta los potenciales efectos secundarios.

Toxicidades como disfunción eréctil, estrechamiento uretral o incontinencia urinaria han estado asociadas la prostatectomía radical. La posibilidad que la RT exacerbe estos síntomas siempre está en mente de médicos y pacientes. La literatura existente indica que la asociación PR+RTE tiene unas tasas de incontinencia similares a la PR exclusiva, aunque son pocos los estudios prospectivos. Recientemente, usando datos recogidos prospectivamente, y con la escala CTCAE v2.0 como referencia, Choo ha evaluado la incidencia y severidad de la toxicidad GU tras RT adyuvante (33). De 74 pacientes estudiados 47 presentaban disfunción GU previa a la RTE, y casi todos ellos (45) incontinencia. Tan alta e inesperada prevalencia puede deberse al poco tiempo transcurrido entre la PR y la RTE. La toxicidad GU pre-RTE estaba significativamente asociada al desarrollo de toxicidad aguda, aunque no hubo ningún caso con incontinencia G3. Incluso los dos pacientes con incontinencia G3 pre-RTE refirieron mejoría post-RTE. El pico en severidad y prevalencia de la incontinencia como efecto agudo estaba en las 10 semanas post-RTE, a diferencia del resto de efectos, situados en torno a las 6 semanas del inicio de la RTE. La incontinencia tardía de importancia tampoco fue destacable. La tasa acumulada de incontinencia $G \geq 2$ era del $13.8 \%$, y sólo un paciente presentaba incontinencia G3 a los 38 meses del tratamiento. En el análisis multivariante, y una vez ajustado por la disfunción previa, sólo la severidad de la toxicidad aguda fue predictora del desarrollo de toxicidad tardía $G \geq 2$.

Otros factores como la edad, la dosis, el intervalo entre la PR y la RTE o la toxicidad GI no fueron determinantes. Es importante hacer notar que, tras 30 meses tras RTE, de 27 pacientes continentes tras la PR, sólo 4 presentaban incontinencia $\mathrm{G} 1$. Y de 40 pacientes con pérdidas pre-RTE, 23 había mejorado y únicamente 2 sufrieron empeoramiento de los síntomas. Exacerbaciones o nuevas manifestaciones de incontinencia son relativamente poco habituales tras RT adyuvante. Estos datos se corroboran con otros estudios, como el de Van Cangh, que no encontró diferencias, a los 24 meses, en la proporción de pacientes continentes tras PR entre los 
que fueron irradiados y los que no (77\% vs.83\%) (34). Otros síntomas, como la urgencia miccional, sí pueden empeorar tras la RTE adyuvante, pero podemos afirmar que la incontinencia severa, y en especial su exacerbación, es fenómeno poco común.

Al hablar de RTE de rescate nos referimos a la persistencia de niveles elevados de PSA post-PR, a la recidiva bioquímica (PSA $>02 \mathrm{ng} / \mathrm{ml}$ ) tras PSA postPR indetectable, o la recidiva histológica. Dada la infraestadificación diagnóstica y el aumento de PR en los últimos años, esta situación no es infrecuente en la práctica diaria.

De nuevo nos vemos obligados a sopesar la QOL post-PR y el posible impacto que en ella tendrá un nuevo y agresivo tratamiento. La mayoría de los estudios en este ámbito tiene dos importantes limitaciones. Suelen ser análisis retrospectivos y carecen de evaluación pre-RTE. El mismo grupo de Choo en 2008 publicó sus datos de toxicidad tras RTE de rescate, recogidos prospectivamente (35). Para ello, al igual que en el estudio de la RT adyuvante usó la escala CTCAE v2.0. El $40 \%$ de los pacientes presentaban síntomas GU previos al tratamiento, la mayoría $(26 / 28)$ debidos a la incontinencia (23 pacientes G1). La presencia de la toxicidad pre-RTE estaba asociada al desarrollo de la toxicidad aguda, aunque el síntoma de mayor prevalencia era la polaquiuria. Una vez finalizado el tratamiento esos síntomas mejoraban, quedando, a las 10 semanas, la incontinencia como síntoma prevalente. Finalmente, de los 26 paciente incontinentes al inicio, 13 de ellos había mejorado a los 30 meses, y sólo dos empeoraron. Y de los 45 pacientes continentes, 14 desarrollaron incontinencia, pero únicamente 1 G2 (uso de pañales). Al igual que con la RTE adyuvante, la incontinencia urinaria severa debida a la RTE es un fenómeno poco común, así como su exacerbación en el caso de su presencia previa.

Otra coincidencia es que la severidad de la toxicidad aguda es predictora de la toxicidad crónica. En cambio, la RTE de rescate, a los 36 meses, presenta una incidencia acumulada de incontinencia $G \geq 2$ ligeramente mayor que la RTE adyuvante.

\section{CONCLUSIONES}

La incontinencia urinaria que precisa del uso de pañales es un acontecimiento adverso poco frecuente tras la RTE. A pesar de ello, y debido al impacto sobre la $Q O L$ de quienes la sufren, identificar a los pacientes con más riesgo de desarrollarla es fundamental. La realización de una RTUP, antes o tras el tratamiento, y la severidad de la toxicidad GU aguda son factores de riesgo. Para profundizar en el estudio de la incontinencia urinaria es necesario un consenso para el uso de cuestionarios y escalas adecuadas, que eviten la pérdida de datos (como ocurre con la incontinencia en la escala RTOG), y permitan comparar las diferentes series. Con este espíritu, el grupo URONCOR, bajo el auspicio de la SEOR, ha creado un registro nacional de cáncer de próstata (RECAP), para recoger retro y prospectivamente los datos de los pacientes tratados con RTE o BT.

\section{BIBLIOGRAFÍA y LECTURAS RECOMENDADAS ( ${ }^{*}$ lectura de interés $y^{* *}$ lectura fundamental)}

**1. D'Amico AV, Whittington R, Malkowicz SB, et al. Biochemical outcome after radical prostatectomy, external beam radiation therapy or interstitial radiation therapy for clinically localized prostate cancer. JAMA, 1998; 280:969-74.

2. Jang TL, Bekelman E, Liu Y, et al. Visits to urologists and radiation oncologists prior to treatment decision making for clinically localized prostate cancer (LCaP). JCO, 2007; 25:S6506.

3. Namiki S, Tochigi T, Kuwahara M, et al. Health related quality of life in Japanese men after radical prostatectomy or radiation therapy for localized prostate cancer. Int J Urol, 2004; 11: 619-627.

4. Penson DF, Feng Z, Kuniyuki A, et al. General quality of life 2 years following treatment for prostate cancer: What influences outcomes? Results from the prostate cancer outcomes study. J Clin Oncol, 2003; 21:1147-1154.

5. Smith DS, Carvalhal GF, Schneider K, et al. Quality-of-life outcomes for men with prostate carcinoma detected by screening. Cancer, 2000; 88:1454-1463.

*6. Chen R, Clark J, Manola J, Talcott JA. Treatment 'mismatch' in early prostate cancer: do treatment choices take patient quality of life into account? Cancer, 2008; 112:61-8.

**7. Ferrer M, Suárez JF, Guedea F, et al. Health-related quality of life 2 years after treatment with radical prostatectomy, prostate brachytherapy, or external beam radiotherapy in patients with clinically localized prostate cancer. Int J Radiat Oncol Biol Phys, 2008; 72:421-32.

*8. Marks LB, Carroll PR, Dugan TC, Anscher MS. The response of the urinary bladder, urethra, and ureter to radiation and chemotherapy. Int J Radiat Oncol Biol Phys, 1995; 31:1257-80.

9. Catarin MV, Manzano GM, Nóbrega Ja, et al. The role of membranous urethral afferent autonomic innervation in the continence mechanism after nerve sparing radical prostatectomy: a clinical prospective study. J Urol, 2008; 180(6):2527-31.

10. Emami B, Lyman J, Brown A, et al. Tolerante of normal tissue to therapeutic irradiation. Int J Ra- 
diat Oncol Biol Phys, 1991; 21:109-122.

11. Grupo ORONCOR-SEOR. Cáncer de próstata. Enfoque multidisciplinar. Ed. MPG. Madrid 2007.

12. Henderson A, Andreyev HJN, Stephens R, Dearnaley D. Health-related quality of life in trials of treatment for early prostate cancer. Considerations for future studies. Clin Oncol, 2006; 18:735-47.

13. Litwin MS, McGuigan KA. Accuracy of recall in health-related quality-of-life assessment among men treated for prostate cancer. J Clin Oncol, 1999; 17(9):2882-8.

14. Cox JD, Stetz J, Pajak TF. Toxicity criteria of the Radiation Therapy Oncology Group (RTOG) and the European Organization for Research and Treatment (EORTC). Int J Radiat Oncol Biol Phys, 1995; 31:1341-6.

15. Pavy JJ, Denekamp J, Letschert J, et al. EORTC Late Effects Working Group. Late effects toxicity scoring: the SOMA scale. Int J Radiat Oncol Biol Phys, 1995; 31:1043-7.

*16. Fontayne V, Villeirs G, Lumen N, De Meerleer G. Urinary toxicity after high dose intensity modulated radiotherapy as primary therapy for prostate cancer. Radiother Oncol, 2009; 92:42-7.

17. Kupelian PA, Buchsbaum JC, Elshaikh MA, Reddy CA, Klein EA. Improvement in relapsefree survival throughout the PSA era in patients with localized prostate cancer treated with definitive radiotherapy: year of treatment an independent predictor of outcome. Int J Radiat Oncol Biol Phys, 2003; 57(3):629-34.

18. Vanuytsel L, Ang KK, Vandenbussche L, et al. Radiotherapy in multiple fractions per day for prostatic carcinoma: late complications. Int J Radiat Oncol Biol Phys, 1986; 12:1589-95.

19. Perez CA, Lee HK, Georgiou A, Lockett MA. Technical factors affecting morbidity in definitive irradiation for localized carcinoma of the prostate. Int J Radiat Oncol Biol Phys, 1994; 28:811-9.

20. Shipley WU, Zietman AL, Hanks GE, et al. Treatment related sequelae following external beam radiation for prostate cancer: a review with an update in patients with stages $\mathrm{T} 1$ and $\mathrm{T} 2$ tumor. $\mathrm{J}$ Urol, 1994; 152:1799-805.

21. Jonler M, Ritter MA, Brinkmann R, Messing EM, Rhodes PR, Bruskewitz RC, et al. Sequelae of definitive radiation therapy for prostate cancer localized to the pelvis. Urology, 1994; 44:876-82.

22. Brabbins D, Martinez A, Yan D, et al. A doseescalation trial with the adaptive radiotherapy process as a delivery system in localized prostate cancer: analysis of chronic toxicity. Int J Radiat Oncol Biol Phys, 2005; 61:400-8.

**23. Zelefsky MJ, Levin EJ, Hunt M, et al. Incidence of late rectal and urinary toxicities after three-dimensional conformal radiotherapy and intensity-modulated radiotherapy for localized prostate cancer.
Int J Radiat Oncol Biol Phys, 2008; 70(4):1124-9.

**24. Liu M, Pickles T, Berthelef E, et al. Urinary incontinente in prostate cancer in patients treated with external beam radiotherapy. Radiother Oncol 2005;74:197-201.

25. Lee WR, Schultheiss TE, Hanlon AL, Hanks GE. Urinary incontinence following external-beam radiotherapy for clinically localized prostate cancer. Urology, 1996; 48:95-9.

26. Sandhu AS, Zelefsky MJ, Lee HJ, et al. Longterm urinary toxicity after 3-dimensional conformal radiotherapy for prostate cancer in patients with prior history of transurethral resection. Int $\mathbf{J}$ Radiat Oncol Biol Phys, 2000; 48:643-7.

27. Green N, Treible D, Wallack H. Prostate cancer: postirradiation incontinence. J Urol 1990; 144:307-9.

28. Patel H, Peddada AV, Zimmern P, Hernandez R, Kagan R. Risk of incontinence with transurethral resection of the prostate after radiation therapy for prostate cancer. J Surg Oncol, 1997; 64:127-9.

29. Holzman M, Carlton CE, Scardino PT. The frequency and morbidity of local tumor recurrence after definitive radiotherapy for stage $\mathrm{C}$ prostate cancer. J Urol 1991;146:1578-82.

30. Liu M, Pickles T, Agranovich A, et al. Impact of neoadjuvant androgen ablation and other factors on late toxicity following external beam prostate radiation. Int J Radiat Oncol Biol Phys, 2004; 58:59-67.

*31. Bolla M, van Poppel H, Collette L, et al. Postoperative radiotherapy after radical prostatectomy: A randomised controlled trial (EORTC trial 22911). Lancet 2005;366:572-578.

32. Wiegel T, Bottke D, Willich N, et al. Phase III results of adjuvant radiotherapy (RT) versus "wait and see" (WS) in patients with pT3 prostate cancer following radical prostatectomy (RP) (ARO 96-02/AUO AP 09/95) [Abstract]. J Clin Oncol, 2005; 23(Suppl. 16S):381s.

*33. Choo R, Pearse M, Danjoux C, et al. Analysis of gastrointestinal and genitourinary morbidity of postoperative radiotherapy for pathologic T3 disease or positive surgical margins after radical prostatectomy using National Cancer Institute Expanded Common Toxicity Criteria. Int J Radiat Oncol Biol Phys, 2008; 72(4):989-995.

34. Van Cangh PJ, Richard F, Lorge F, et al. Adjuvant radiation therapy does not cause urinary incontinence after radical prostatectomy: Results of a prospective randomized study. J Urol, 1998; 159:164-166.

*35. Pearse M, Choo R, Danjoux C, et al. Prospective assessment of gastrointestinal and genitourinary toxicity of salvage radiotherapy for patients with prostate-specific antigen relapse or local recurrence after radical prostatectomy. Int J Radiat Oncol Biol Phys, 2008; 72(3):792-8. 